\title{
Targeted transplantation of iron oxide-labeled, adipose-derived mesenchymal stem cells in promoting meniscus regeneration following a rabbit massive meniscal defect
}

\author{
YIYING QI, ZHIGAO YANG, QIANHAI DING, TENGFEI ZHAO, ZHONGMING HUANG and GANG FENG \\ Department of Orthopedic Surgery, Second Affiliated Hospital, Zhejiang University School of Medicine, \\ Hangzhou, Zhejiang 310009, P.R. China
}

Received January 31, 2015; Accepted November 25, 2015

DOI: $10.3892 /$ etm.2015.2944

\begin{abstract}
Repair of a massive meniscal defect remains a challenge in the clinic. However, targeted magnetic cell delivery, an emerging technique, may be useful in its treatment. The present study aimed to determine the effect of targeted intra-articular injection of superparamagnetic iron oxide (SPIO)-labeled adipose-derived mesenchymal stem cells (ASCs) in a rabbit model of a massive meniscal defect. ASCs may be directly labeled and almost $100 \%$ of the ASCs were labeled with SPIO after $24 \mathrm{~h}$; these SPIO-labeled ASCs may be orientated by magnet. The centrifuged SPIO-labeled ASCs precipitations may be detected by magnetic resonance imaging (MRI). The anterior half of the medial meniscus of 18 New Zealand Rabbits was excised. After 7 days, the rabbits were randomized to injections of $2 \times 10^{6}$ SPIO-labeled ASCs, $2 \times 10^{6}$ unlabeled ASCs or saline. Permanent magnets were fixed to the outside of the operated joints for one day, and after 6 and 12 weeks, the knee joints were examined using MRI, gross and histological observation, and Prussian blue staining. Marked hypointense artifacts caused by SPIO-positive cells in the meniscus were detected using MRI. Histological observation revealed that the anterior portion of the meniscus was similar to the native tissue, demonstrating typical fibrochondrocytes surrounded by richer extracellular matrix in the SPIO-ASCs group. Collagen-rich matrix bridging the interface and the neo-meniscus integrated well with its host meniscus. Furthermore, degenerative changes occurred in all groups, but intra-articular injection of SPIO-ASCs or ASCs alleviated these degenerative changes. Prussian blue staining indicated that the implanted ASCs were directly associated with the
\end{abstract}

Correspondence to: Dr Gang Feng, Department of Orthopedic Surgery, Second Affiliated Hospital, Zhejiang University School of Medicine, 88 Jiefang Road, Hangzhou, Zhejiang 310009, P.R. China E-mail:fngn@sina.com

Key words: adipose-derived mesenchymal stem cells, superparamagnetic iron oxide, meniscus, targeted intra-articular delivery regenerated tissue. Overall, targeted intra-articular delivery of SPIO-ASCs promoted meniscal regeneration whilst providing protective effects from osteoarthritic damage.

\section{Introduction}

The meniscus has important roles in joint stability, shock absorption and load distribution (1-3). Injuries to the meniscus are a common source for knee dysfunction and disability due to the limited self-repair capacity of the meniscus (4-6). For symptomatic meniscus injury, a total or partial meniscectomy is often performed. However, loss of meniscal tissue often leads to long-term degenerative joint changes, articular cartilage degeneration and eventually, osteoarthritis (OA) $(7,8)$. A novel strategy for meniscus repair is required, and the less invasive cell-based therapy for meniscus regeneration is a promising possibility.

The availability of large quantities of mesenchymal stem cells (MSCs), which may be obtained from the majority of adult tissues, and their multipotency, specifically their chondrogenic differentiation properties, make MSCs the most suitable candidate progenitor cell source for cell-based therapy. Fraser et al (9) reported that the frequency of MSCs in adipose tissue is $~ 500$-fold more than that in bone marrow. Adipose-derived mesenchymal stem cells (ASCs) are therefore a promising candidate cell source for meniscus regeneration through the less invasive technique of intra-articular injection.

The ability to monitor the in vivo behavior of implanted MSCs and understand their fate is important for developing successful cell therapies; an effective, non-invasive and non-toxic technique for cell tracking is required for this purpose (10). Superparamagnetic iron oxide (SPIO) is an ideal tracer for MSCs, which may be labeled with SPIO at $>90 \%$ efficiency without the use of a transfection agent $(11,12)$. The SPIO-labeled MSCs may then be visualized by magnetic resonance imaging (MRI). The success of cell therapies depends on the ability to deliver the cells to the site of injury and targeted magnetic cell delivery is a promising emerging technique for localized cell transplantation therapy.

In the present study, SPIO was used to label ASCs. A permanent magnet close to the joint was used to localize 
the implanted ASCs. The effect and outcome of the targeted intra-articular injection of SPIO-labeled ASCs in a rabbit model of massive meniscal defect were then determined. These results enabled investigation of meniscal regeneration, OA prevention and the destination of the ASCs.

\section{Materials and methods}

Cell isolation and culture. The ASCs were isolated from the subcutaneous fat of the nape of the neck of rabbits using methods described previously (13). The experimental procedures used were approved by the Experimental Animal Ethics Committee of Zhejiang University (Hangzhou, China). The isolated tissues were homogenized in Dulbecco's modified Eagle's medium (DMEM) and digested in 0.1\% type I collagenase (Sigma-Aldrich, St. Louis, MO, USA) at $37^{\circ} \mathrm{C}$ for $1 \mathrm{~h}$ with intermittent shaking. Cells were collected following centrifugation at $1,200 \mathrm{x}$ g for $10 \mathrm{~min}$, cultured in DMEM supplemented with $10 \%$ fetal bovine serum, $100 \mathrm{U} / \mathrm{ml}$ penicillin and $100 \mathrm{mg} / \mathrm{ml}$ streptomycin (Thermo Fisher Scientific, Inc., Waltham, MA, USA), and incubated at $37^{\circ} \mathrm{C}$ in $5 \% \mathrm{CO}_{2}$. The first medium change was performed $24 \mathrm{~h}$ after plating and once every 2-3 days routinely. The cells were harvested or subcultured as they reached $80-90 \%$ confluency. Cells were used for the following experiments at the third passage.

SPIO labeling and Prussian blue staining. For incubation with SPIO, Ferucarbotran (Bayer Schering Pharma AG, Berlin, Germany) was added to the culture medium at concentrations of $0,1,10$ and $100 \mu \mathrm{g} \mathrm{Fe} / \mathrm{ml}$ in accordance with previous direct labeling studies $(14,15)$. After $24 \mathrm{~h}$, the ASCs treated with $100 \mu \mathrm{g} \mathrm{Fe} / \mathrm{ml}$ Ferucarbotran were stained with Prussian blue. The ASCs were treated with $10 \%$ potassium ferrocyanide and $20 \%$ hydrochloric acid for $20 \mathrm{~min}$. ASCs were then washed twice with double-distilled water and visualized under an IX71 light microscope (Olympus Corporation, Tokyo, Japan).

Cell proliferation. SPIO-treated ASCs at concentrations of 1, 10 and $100 \mu \mathrm{g} \mathrm{Fe} / \mathrm{ml}$, and unlabeled cells, were grown in 96-well plates at $1 \times 10^{4}$ cells/well for $24 \mathrm{~h}$. The proliferation was evaluated by 3-(4,5-dimethyl-2-thiazolyl)-2,5-diphenyl-2H-terazolium bromide (MTT) assay (Sigma-Aldrich). This assay is based on the ability of mitochondrial dehydrogenases to oxidize MTT, a tetrazolium salt, into an insoluble blue formazan product. The optical density of the plates was then read using a Model 550 microplate reader (Bio-Rad Laboratories, Inc., Hercules, CA, USA) using test and reference wavelengths of $570 \mathrm{~nm}$. This test was repeated 3 times.

In vitro cellular MRI. To determine the sensitivity of the MRI magnets for the detection of labeled cells, labeled and unlabeled ASCs were trypsinized, centrifuged at $800 \mathrm{x} \mathrm{g}$ for $5 \mathrm{~min}$ and placed in Eppendorf tubes $\left(5 \times 10^{5}, 1 \times 10^{5}\right.$ and $5 \times 10^{4}$ labeled cell precipitations in $2 \mathrm{ml}$ culture solutions). In vitro cellular imaging was performed with a clinical 3.0-T MR imager using a T2*-weighted gradient-echo (GRET2-WI). Sequence parameters were as follows: Repetition time (TR), $600 \mathrm{msec}$; echo time (TE), $18 \mathrm{msec}$; field of view (FOV), 160x160; flip angle, $40^{\circ}$; matrix size, $512 \times 512$; slice thickness, $2.0 \mathrm{~mm}$; and slice, $0.5 \mathrm{~mm}$.
Meniscectomy and MSC injection. A total of 18 New Zealand 12 week-old rabbits, weighing $2.5 \pm 3.0 \mathrm{~kg}$, were used. The procedures were followed in accordance with the standards described in the 8th edition of the Guide for the Care and Use of Laboratory Animals. Following anesthetization with $30 \mathrm{mg} / \mathrm{kg}$ sodium pentobarbital (3\%; Sangon Biotech Co., Ltd., Shanghai, China) via intravenous injection, a straight incision was made on the anterior side of the bilateral knee and the anteromedial side of the joint capsule, and the anterior horn of the medial meniscus was dislocated anteriorly with forceps (Fig. 1A). The meniscus was then incised vertically at the level of the medial collateral ligament, and the anterior half of the medial meniscus was excised (Fig. 1B). The dislocated meniscus was removed and the wound was closed in layers. After 7 days, $2 \times 10^{6}$ ASCs $(n=6)$ or $2 \times 10^{6}$ SPIO-labeled ASCs $(\mathrm{n}=6)$ in $100 \mu \mathrm{l}$ phosphate-buffered saline (PBS) were injected into the knee joint via a medial approach with a 26-gauge needle. For the control group, the same volume of PBS (only) was injected into the knee $(n=6)$. Following surgery, permanent magnets were fixed to the outside of the treated joints for one day and the rabbits were allowed to walk freely in the cage.

In vivo MRI. The rabbits were imaged under general anesthesia at 6 and 12 weeks post-injection, and MR images of the rabbit joints were obtained on a clinical 3.0-T MR imager equipped with a coil. Sagittal and axial images were obtained using a GRET2-WI sequence. Imaging parameters were as follows: TR, $600 \mathrm{msec}$; TE, $18 \mathrm{msec}$; FOV, 160x160; flip angle, $40^{\circ}$; matrix size, $512 \times 512$; slice thickness, $2.0 \mathrm{~mm}$; and slice, $0.5 \mathrm{~mm}$.

Gross observation. The rabbits were sacrificed at 6 and 12 weeks after surgery. Macroscopic observations of the meniscus, tibial plateau and surrounding joint tissues were performed, in accordance with the International Cartilage Repair Society (ICRS) cartilage repair assessment system $(16,17)$. Using this system, the assessment was recorded using macroscopic examination on the surface of the cartilage. Overall repair assessment was scored and later graded, from normal (grade I) to severely abnormal (grade IV).

Histological observation. Following gross examination, the samples were fixed in $4 \%$ formalin, embedded in paraffin and sliced into $7-\mu \mathrm{m}$ sections. The sections were stained with hematoxylin and eosin for morphological evaluations and with Safranin for glycosaminoglycan distribution, and examined under an IX71 light microscope.

Tracking of SPIO-labeled ASCs. The sections of tissue from the SPIO-labeled ASC group were stained with Prussian blue to detect whether implanted ASCs were involved in meniscal regeneration. Surrounding joint tissues were also fixed in $4 \%$ formalin, embedded in paraffin and sliced into $7-\mu \mathrm{m}$ sections, which were stained with Prussian blue to track the mobilization of implanted ASCs.

Statistical analysis. Results were analyzed by one-way analysis of variance using SPSS 15.0 software (SPSS, Inc., Chicago, IL, USA). Tukey's test was used for multiple comparisons, and the level of significance was set at $\mathrm{P}<0.05$. 

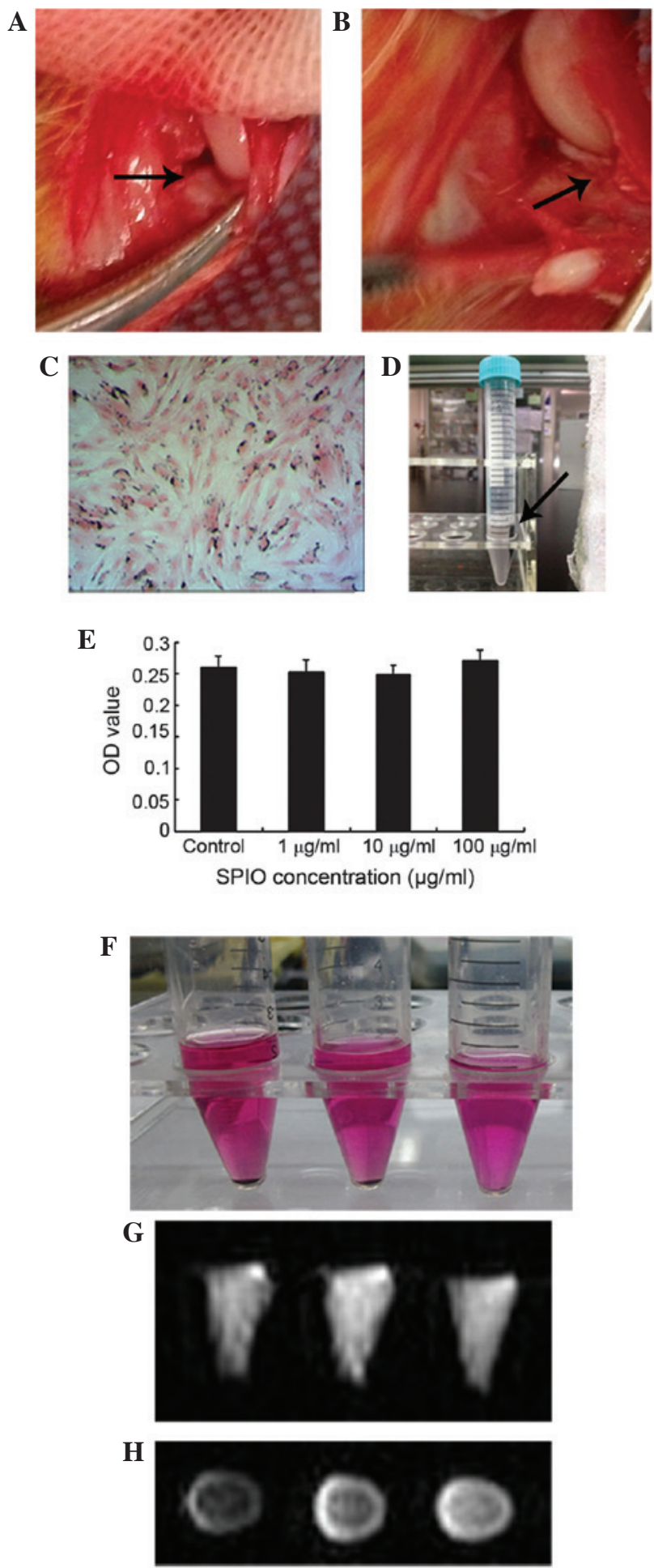

Figure 1. Surgical procedure for massive meniscectomy. (A) The media meniscus was anteriorly dislocated, as indicated by the arrow. (B) The anterior half of the meniscus was excised, and tibial cartilage was exposed, as indicated by the arrow. (C) Prussian blue staining of SPIO-labeled ASCs (magnification, $\mathrm{x} 40$ ). Several blue granules were evident in the cytoplasm surrounding the nuclei. (D) Labeled ASCs could be orientated to the direction of the magnet, as indicate by the arrow. (E) Assessment of cell proliferation. Ferucarbotran-treated and untreated cells were cultured for $24 \mathrm{~h}$. Cell proliferation was measured by MTT assay. No statistical difference in proliferation was evident between untreated cells and cells treated with 1-100 $\mu \mathrm{g} \mathrm{Fe} / \mathrm{ml}$ SPIO. (F) Centrifuged SPIO-labeled ASCs were precipitated in the bottom of test tubes. ( $\mathrm{G}$ and $\mathrm{H}$ ) Gradient-echo $\mathrm{T} 2{ }^{*}$-weighted magnetic resonance images of the three tubes. From left to right: $5 \times 10^{5}, 1 \times 10^{5}$ and $5 \times 10^{4}$ SPIO-labeled ASCs. SPIO, superparamagnetic iron oxide; ASCs, adipose-derived mesenchymal stem cells; OD, optical density.
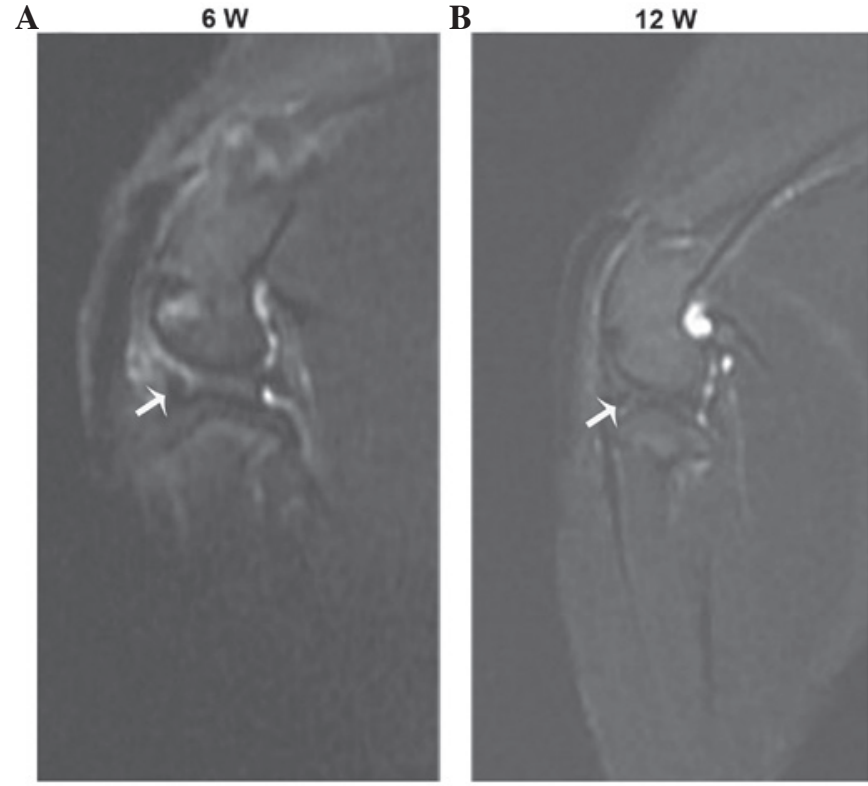

Figure 2. Sagittal gradient-echo $\mathrm{T} 2{ }^{*}$-weighted magnetic resonance images of the knee in the SPIO-ASCs group obtained at (A) 6 and (B) 12 weeks after injection. Low signal intensity indicates the presence of SPIO-labeled ASCs. Arrows indicate an SPIO-positive area, demonstrating that SPIO-labeled cells were present in the meniscus. SPIO, superparamagnetic iron oxide; ASCs, adipose-derived mesenchymal stem cells.

\section{Results}

Cell labeling. ASCs may be directly labeled with SPIO. After $24 \mathrm{~h}$, nearly $100 \%$ of the ASCs were labeled with SPIO, as evidenced by Prussian blue staining (Fig. 1C); SPIO particles stained as blue granules. The blue granules were only observed in the cytoplasm and were predominantly arranged around the nucleus. However, there was no morphological change following SPIO labeling.

Cell proliferation. The labeled ASCs may be orientated in the direction of the magnet (Fig. 1D). No statistically significant differences in cell viability were detected between the SPIO-treated and untreated ASCs, as determined by MTT assay (Fig. 1E).

Cellular MRI of SPIO. After $24 \mathrm{~h}$ of incubation with Ferucarbotran $(100 \mu \mathrm{g} / \mathrm{ml})$, the ASCs were centrifuged; $5 \times 10^{4}$, $1 \times 10^{5}$ and $5 \times 10^{4}$ SPIO-labeled cell precipitations in $2 \mathrm{ml}$ medium were placed in test tubes (Fig. 1F). Under T2-weighted MRI, the centrifuged, labeled cell precipitations in the test tubes were imaged (Fig. $1 \mathrm{G}$ and $\mathrm{H}$ ). However, the centrifuged $1 \times 10^{4}$ SPIO-labeled cell precipitation was not detected by MRI (data not shown).

In vivo MR tracking of MSCs. At 6 and 12 weeks after implantation, clear hypointense artifacts, caused by SPIO-positive cells in the meniscus, were detected using MRI (Fig. 2A and B). However, the intensity of these hypointense signals decreased between 6 and 12 weeks.

Macroscopic observation. All animals were mobile 1-2 $\mathrm{h}$ after surgery and there were no instances of infection. The animals 

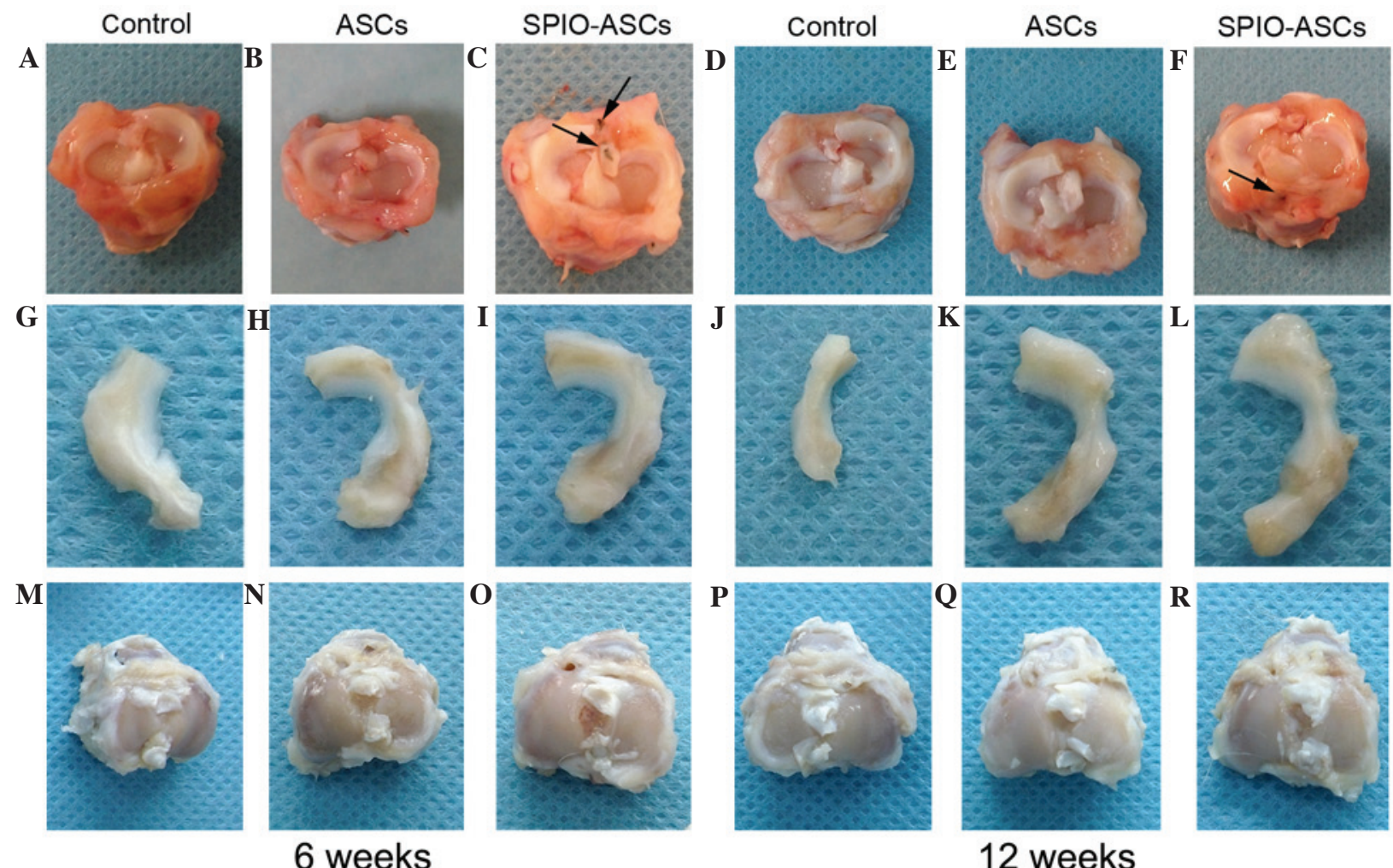

6 weeks
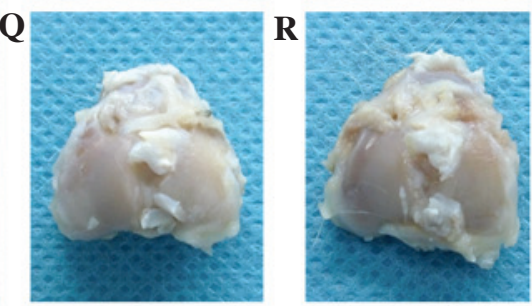

12 weeks

Figure 3. Representative tibial joint, regenerated meniscus and joint surface of tibia at 6 and 12 weeks after surgery. Arrows indicate an SPIO-positive area. A-F: Representative tibial joint in each group; G-L: representative regenerated meniscus in each group; M-R: representative surface of tibia in each group. ASCs, adipose-derived mesenchymal stem cells; SPIO, superparamagnetic iron oxide; H\&E, hematoxylin and eosin.

tolerated the cell injection well, and there was no evidence of local inflammation, immobilization or unloading of the joint.

Fig. 3 demonstrates representative images of the post-surgery tibial joint, regenerated meniscus and joint surface of the tibia. At 6 weeks, the anterior section of the meniscal defect of the ASC and SPIO-ASC injection groups exhibited improved meniscal regeneration in contrast to the control group (Fig. 3A-C and G-I). The SPIO-ASCs group reported the highest level of meniscal regeneration and a good meniscal shape. In addition, SPIO-positive staining was observed in the surrounding tissues (Fig. 3C).

At 12 weeks, in the control group, the meniscal tissue had deteriorated (Fig. 3D and J). The majority of the meniscuses had regenerated in the ASC group (Fig. 3E and $\mathrm{K}$ ) and the SPIO-ASCs group reported almost normally-shaped meniscuses (Fig. 3F and L). SPIO-positive staining was also reported in the surrounding tissues of the SPIO-ASCs group (Fig. 3F).

Protection from osteoarthritic damage. Besides meniscal regeneration, degenerative changes, including cartilage erosion, and osteophyte formation on the surface of the medial condyle of femur and medial tibial plateau, were evaluated (Fig. 3). The degenerative changes on the surface of the tibial plateau were more severe than those of the condyle of the femur, which was less affected by meniscal degeneration, in all groups. From 6-12 weeks, the degenerative changes were more severe in the control group; in this group, a large region of osteophytes developed on the surface of the tibial plateau (Fig. 3A and G). In the ASC- and SPIO-ASC-treated joints, the degree of cartilage destruction and osteophyte formation were all markedly reduced compared with that of the control joints (Fig. 3B, C, H and I). At 12 weeks, based on the ICRS grading system, in the control group, 1 knee had grade I lesions, 3 knees had grade II lesions and 2 knees had grade III lesions. By contrast, in the ASC-treated group, 2 knees had grade 0 lesions and 4 knees had grade I lesions. In the SPIO-ASC-treated group, 3 knees had grade 0 lesions and 3 knees had grade I lesions. All results indicated that ASC and SPIO-ASC transplantation had the overall effect of protection from OA.

Histological observation. At 6 weeks post-implantation, the control group demonstrated regeneration of more fibroblast-like tissue and less hypercellular fibrocartilaginous tissue at the anterior portion of the meniscus (Fig. 4A, D, G and J). The anterior portion of the meniscus was partially repaired by hypercellular fibrocartilaginous tissue and less fibrous-like tissue in the ASC group (Fig. 4B, E, H and K). In the SPIO-ASC group, the anterior portion of the meniscus was regenerated, with a greater mass of hypercellular fibrocartilaginous tissue and extracellular matrix (ECM) (Fig. 4C, F, I and L).

At 12 weeks post-implantation, the regenerated meniscus deteriorated in the control group, and was filled with fibroblastic cells and reduced extracellular matrix; the edge of this region was also atrophied (Fig. 5A, D, G and J).

In the ASC group, the anterior portion of the meniscus was occupied by hypercellular fibrocartilaginous tissue with a low quantity of ECM. However, the neomeniscus was not normally shaped (Fig. 5B, E, H and K). In the SPIO-ASC group, the anterior portion of the meniscus was similar to 


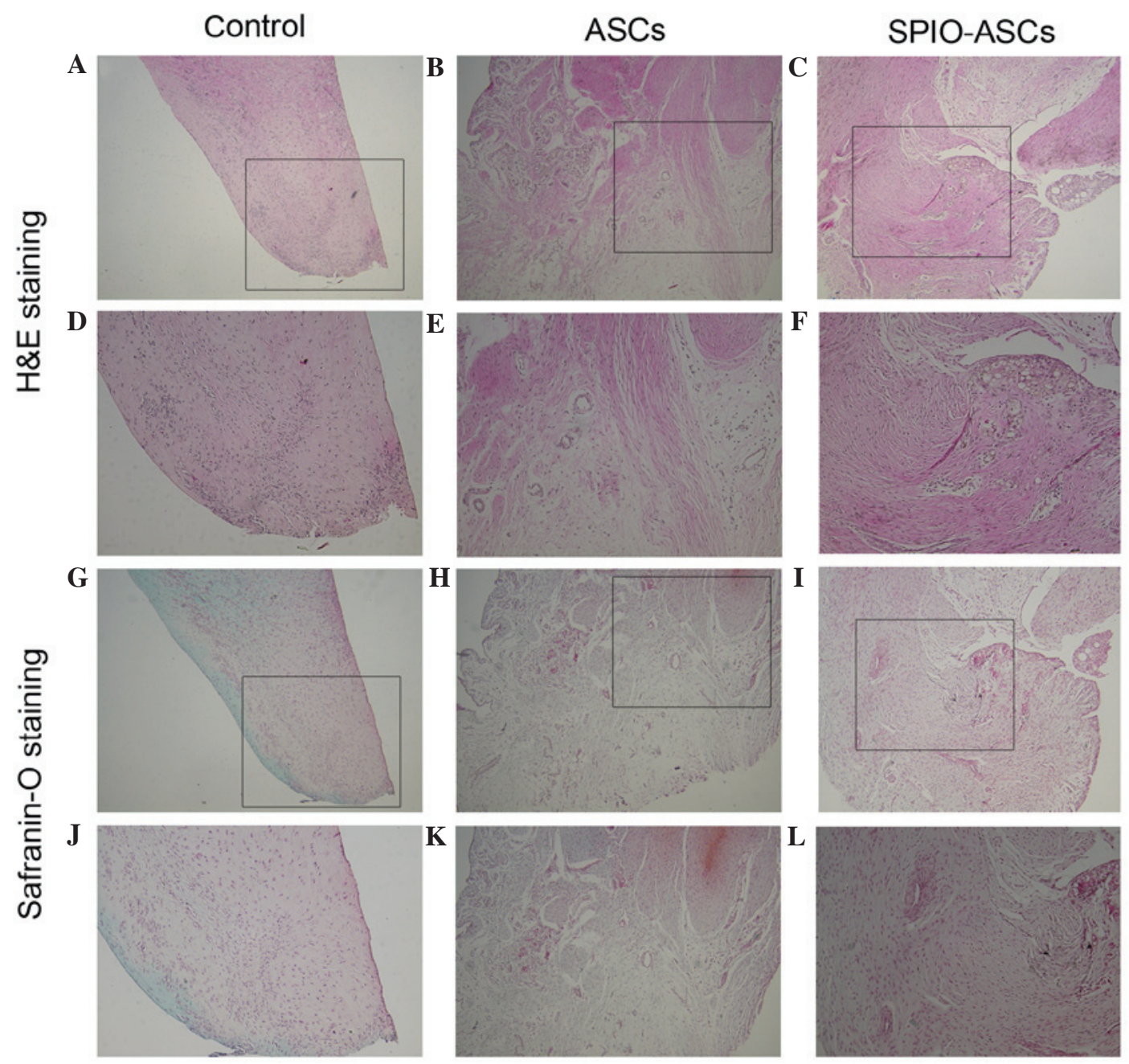

Figure 4. Histological observation of regenerated meniscal tissues at 6 weeks post-injection. ASCs, adipose-derived mesenchymal stem cells; SPIO, superparamagnetic iron oxide; H\&E, hematoxylin and eosin. (A-C, G-I) Magnification, x40. (D-F, J-L) Magnification, x100.

the native tissue, demonstrating typical fibrochondrocytes surrounded by collagen-rich ECM that bridged the interface; the neomeniscus also integrated well with the host meniscus (Fig. 5C, F, I and L). Scar tissue was not observed in any groups at any time point.

ASC grafting. Prussian blue staining of sections of neomeniscal tissue in each sample indicated that the implanted ASCs were directly associated with the regenerated tissue (Fig. 6A-I). SPIO-positive cells were detected in all five samples at 6 weeks (Fig. 6A-E) and in four of five samples at 12 weeks (Fig. 6F-I). The SPIO-positive cells were typically associated with the edge of the tissue and, in numerous cases, were also detected in the interior of the tissue. A number of typical fibrochondrocytes were stained with Prussian blue, indicating that implanted ASCs differentiated directly into fibrochondrocytes and contributed to meniscus regeneration (Fig. 6E). The injected ASCs also colonized and integrated into the surface layers of soft tissues within the joint, including the synovial capsule, fat tissue and posterior cruciate ligament at 6 and 12 weeks post-injection (Fig. 6J-Q). The synovial capsule, fat tissue and posterior cruciate ligament demonstrated a high incidence of SPIO-positive cells. However, evidence of cell grafting onto articular cartilage from the femoral condyles and the tibial plateaus was not detected.

\section{Discussion}

The present study demonstrated that targeted intra-articular delivery of SPIO-ASCs promoted meniscus regeneration whilst providing protection from osteoarthritic damage, unaffected by SPIO labeling; this is likely to be attributable to paracrine effects or in situ modulation of the healing response. Furthermore, the implanted SPIO-labeled ASCs adhered to the injured meniscus and were directly associated with meniscal regeneration, observed using Prussian blue staining and MRI.

SPIO has been widely used for stem cell labeling (10). The present results revealed that ASCs may be efficiently labeled with $100 \mu \mathrm{g} / \mathrm{ml}$ SPIO without using a transfection agent; the labeling efficiency was almost $100 \%$ after a $24-\mathrm{h}$ incubation, which was consistent with previous studies $(15,18)$. Using transfection agents may increase intracellular SPIO uptake; however, transfection agents may have adverse effects on MSCs. Arbab et al (19) investigated the effects of ferumoxides with various transfection agents on MSCs; following an increase in iron concentration from 50 to $125 \mu \mathrm{g} / \mathrm{ml}$, the MSC 

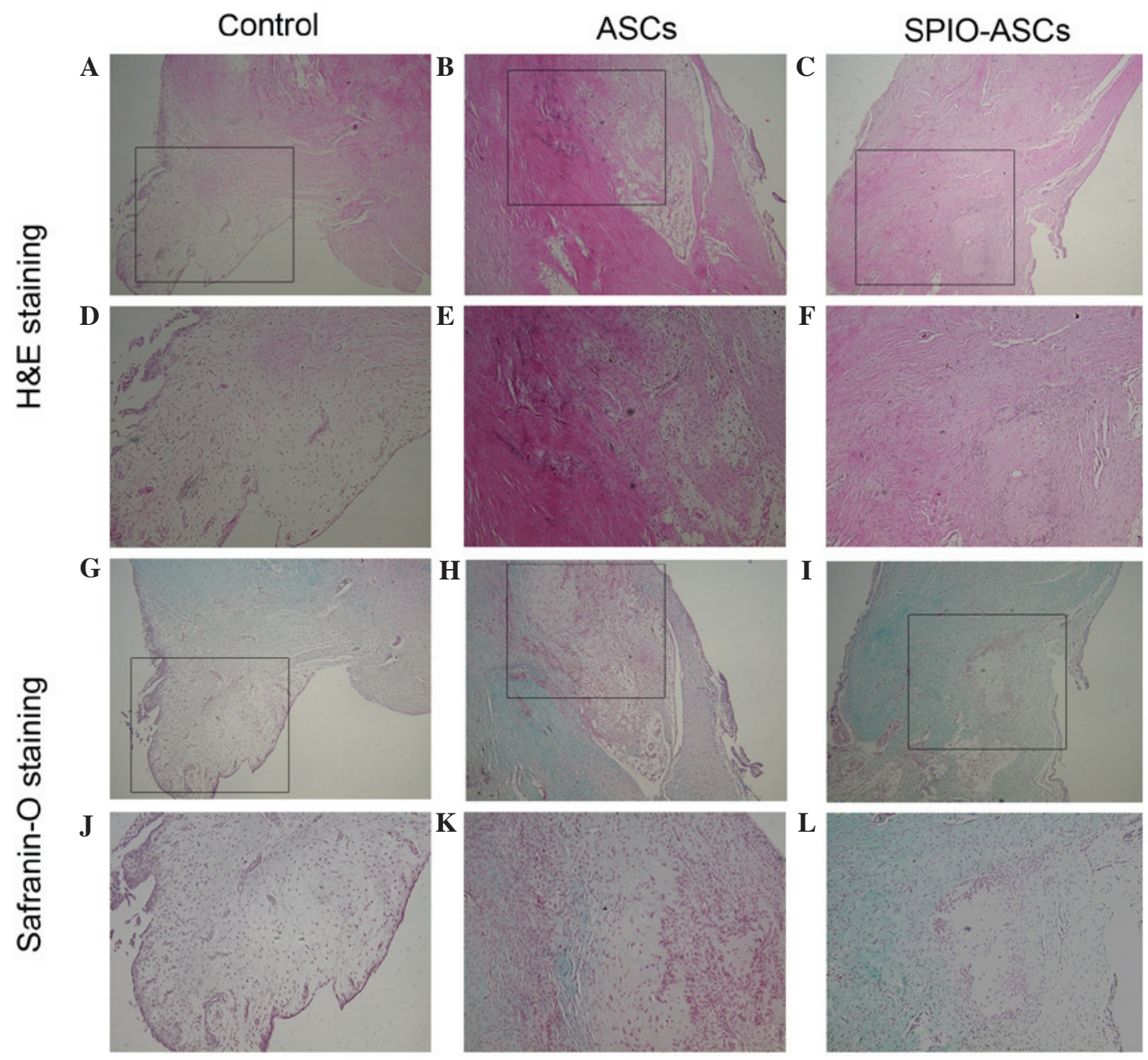

Figure 5. Histological observation of regenerated meniscal tissues at 12 weeks after injection. MSCs, mesenchymal stem cells; SPIO, superparamagnetic iron oxide; H\&E, hematoxylin and eosin; ASCs, adipose-derived mesenchymal stem cells. (A-C, G-I) Magnification, x40. (D-F, J-L) Magnification, x100.

intracellular iron concentration demonstrated a 3 -fold rise after $24 \mathrm{~h}$, leading to $\sim 40 \%$ cell mortality when compared with unlabeled MSCs. These results suggest that a higher intracellular free iron concentration may be toxic to the cells and that an iron concentration safety threshold exists. This toxic effect may be due, in part, to the transfection agents. Direct SPIO labeling of cells without transfection agents would therefore advance the clinical use of these particles $(14,20)$. The direct iron uptake of the cells could reach an amount comparable to that of SPIO labeling with a transfection agent by increasing SPIO concentration (14), which can be observed in the present study. In the current study, ASCs were incubated in SPIO at concentrations up to $100 \mu \mathrm{g} \mathrm{Fe} / \mathrm{ml}$ of ferucarbotran for $24 \mathrm{~h}$ to achieve appropriate and optimized cell labeling. A previous study has reported that cell viability was not significantly affected following incubation with high ferucarbotran concentrations of $>500 \mu \mathrm{g} \mathrm{Fe} / \mathrm{ml}$ (21). In the present study, ASCs were labeled with numerous concentrations of SPIO and no significant difference was identified in the proliferation of ASCs between these groups. Furthermore, another previous study revealed that direct labeling with ferucarbotran did not impair the function or toxicity, or inhibit the differentiation capacity of MSCs (15).
MSCs are multipotent cells and have been demonstrated to effect positive roles in cartilage regeneration (22). MSCs have demonstrated their ability to migrate and graft onto the site of injury, and undergo site-specific differentiation (23). MSCs may frequently be obtained from adipose tissue (24). The present in vivo study revealed that direct intra-articular delivery of ASCs markedly improved the meniscus regeneration compared with an untreated group. This method creates a possibility of regenerating the meniscus with less invasiveness when compared with meniscal transplantation. Previous studies described that the injection of MSCs from different sources into the joint contributed to meniscus regeneration (25-27), concordant with the results of the current study.

A massive meniscal defect would inevitably lead to OA in the long term. In the present study, all groups succumbed to OA. However, intra-articular ASCs can provide a protective effect from osteoarthritic damage compared with the control group. Stem cells represent a possibility for relief of the tumor burden in degenerative joint diseases via direct intra-articular injection (28). Stem cells are known to produce numerous, diverse bioactive molecules with immunoregulatory $(29,30)$ and/or regenerative functions (31). Through direct cell-cell interaction or the secretion of a variety of factors, stem cells may exert a 


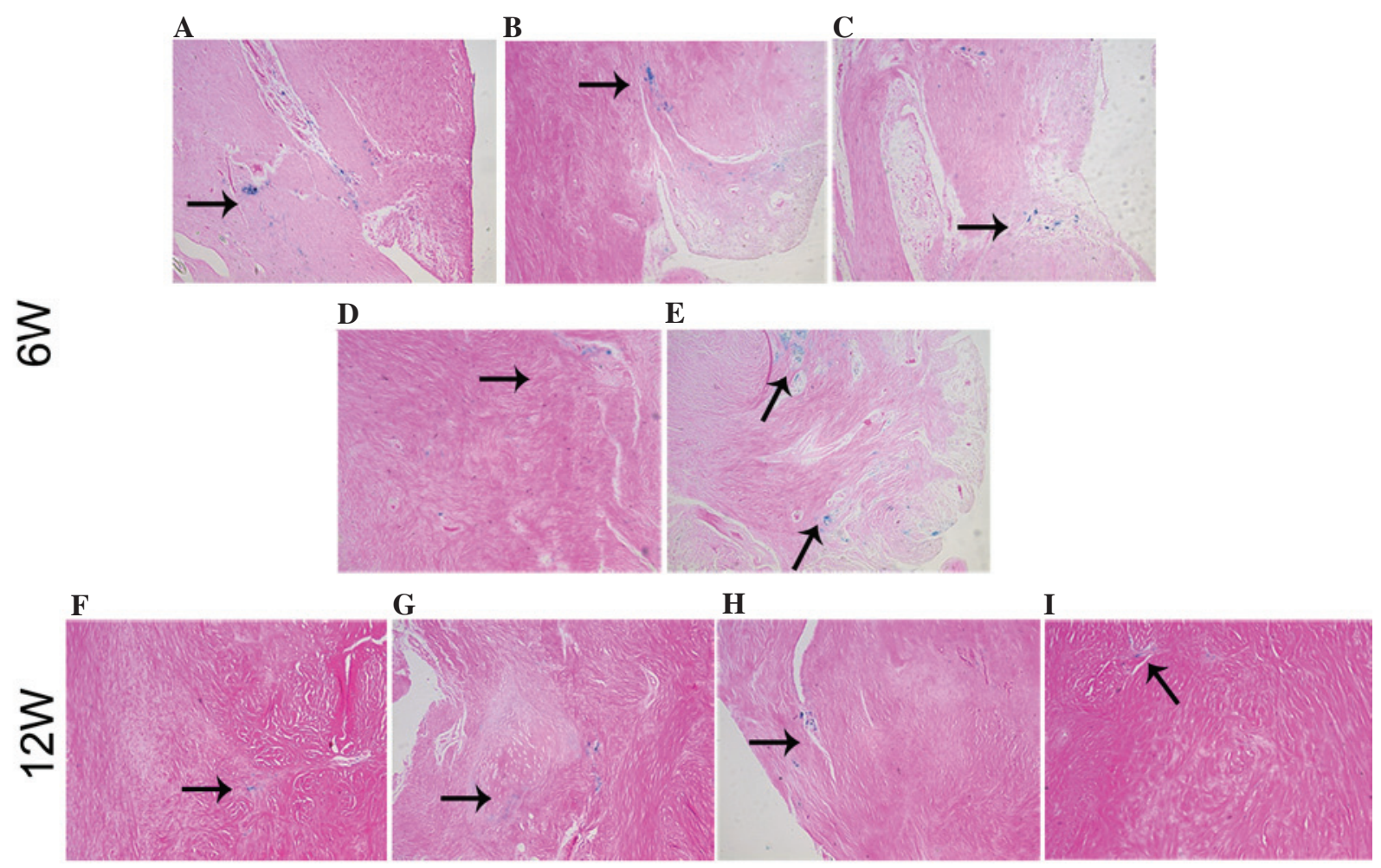

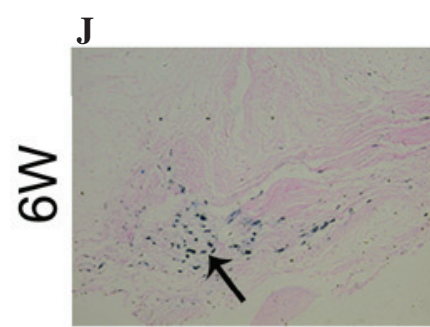

K
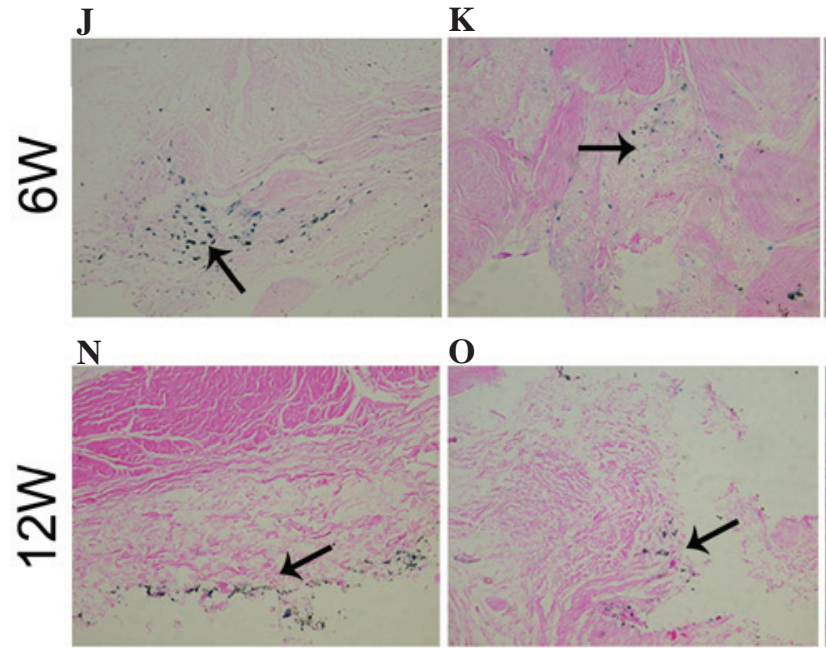

$\mathbf{0}$

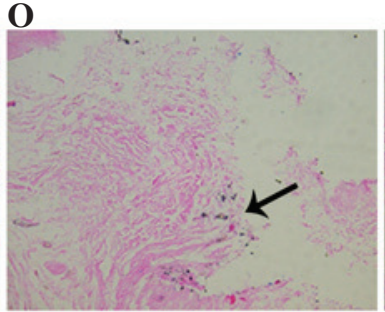

L

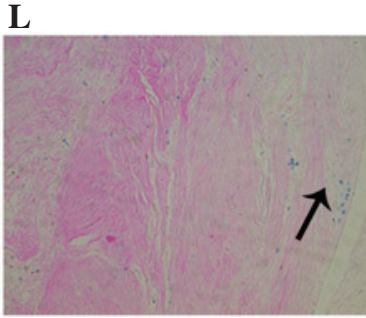

$\mathbf{P}$

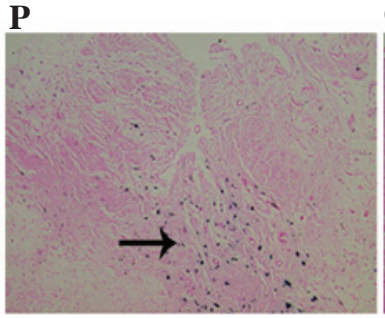

M

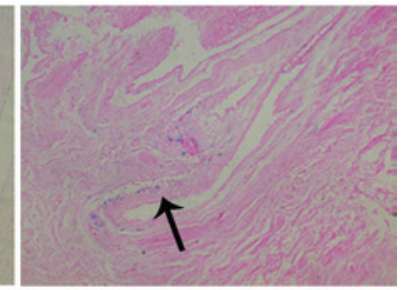

Q

Figure 6. Prussian blue staining of the regenerated meniscus in each sample and surrounding host tissue at 6 and 12 weeks post-injection Arrows indicate SPIO-positive cells. (A-I) SPIO-positive cells were detected primarily at the surface and in the center of neomeniscal tissue. (J-Q) Synovial capsule, fat tissue and posterior cruciate ligament showed a high incidence of SPIO-positive cells. SPIO, superparamagnetic iron oxide. Magnification, x100.

marked effect on local tissue repair through modulation of the local microenvironment and activation of endogenous progenitor cells (32). Stem cells, therefore, not only have the ability to contribute structurally to tissue repair, but also possess potent immunomodulatory and anti-inflammatory effects; stem cells thereby provide protection from osteoarthritic damage, as observed in the ASCs and SPIO-ASCs groups of the present study.

Cell migration and/or differentiation are necessary for endogenous meniscal healing and repair. The implanted stem cells adheres to the meniscal defect first, and subsequently induce the chondrogenic differentiation of adhered stem cells, which may be more useful for meniscal repair. Stem cells have revealed an ability to migrate and graft onto multiple musculoskeletal tissues, particularly sites of injury, and to undergo site-specific differentiation (23). However, in-site migration is much less prevalent. In the present study, under an external magnetic force, SPIO-labeled ASCs were successfully targeted and adhered to the meniscal defect and then differentiated into fibrochondrocytes. The regenerated meniscus was similar to the native tissue, demonstrating typical fibrochondrocytes surrounded by collagen-rich ECM. Due to the paracrine and trophic effects of ASCs, the SPIO-ASC group reported the highest level of meniscus regeneration and less osteoarthritic damage.

Monitoring implanted ASCs is vital to successful cell-based therapies. MRI provides a non-invasive method for studying the fate of transplanted cells labeled with SPIO, and allows imaging at the cellular and molecular levels (33). The MRI signal 
intensity is directly associated with the amount of intracytoplasmatic SPIO in the surviving cells. In the present study, in vitro, centrifuged, labeled ASC precipitations in test tubes were visualized using high-resolution MRI. In vivo, the implanted ASCs were successfully tracked by MRI, and Prussian blue staining indicated that the ASCs not only adhered to the meniscal defect, but also to other structures, including the synovial capsule, fat tissue and the anterior cruciate ligament. Use of MRI, combined with histological observation, may accurately confirm the location of implanted SPIO-labeled ASCs. However, whether the ASCs additionally grafted to distant organs was not detected. A previous study demonstrated that the intra-articularly injected MSCs remained in the knee joint and did not migrate to other organs (25). However, subsets of the implanted ASCs migrated to untargeted sites in the current study. A method by which to effectively guide the stem cells to the targeted sites therefore requires additional study. SPIO-labeled MSC-based targeted therapy has the potential for treating human diseases. Either a magnetic field or MRI may be used to guide and monitor SPIO-labeled MSCs to target organs. Furthermore, the dilution of iron oxide particles following cell proliferation, migration of labeled cells, and biodegradation of iron oxide particles may decrease the intensity of hypointense signals (19), which may limit the duration that MSCs could be accurately detected by MRI.

Despite a high degree of cell loss, labeled and unlabeled MSCs provide a possible therapeutic benefit in meniscal regeneration and OA prevention. The therapeutic effects of transplanted cells may be independent of implanted cell survival or transdifferentiation. Furthermore, factors secreted by the MSCs (paracrine effect) or an alternative association with the healing response may have a beneficial effect in meniscal regeneration.

The targeting efficiency was not high in the present study, demonstrating that SPIO-ASCs also migrated to other, untargeted tissues. Additional studies are required to investigate novel methods in order to improve the targeting of SPIO-ASCs. Magnetic resonance targeting (MRT) is a promising method for the magnetic targeting of deep organs; MRT may steer SPIO-labeled cells to their target region by means of magnetic field gradients inherent to all MRI systems. It remains uncertain whether the regenerated meniscus functions in a normal manner and prevents secondary osteoarthritic change in the long term. Future studies should, therefore, include biomechanical and biochemical analysis of the regenerated meniscus.

In conclusion, in the present study, the targeted intra-articular delivery of SPIO-ASCs promoted meniscus regeneration whilst providing protective effects from osteoarthritic damage. SPIO-labeled ASCs injected into the massive meniscectomized knee were successfully and non-invasively tracked by MRI. In addition, implanted ASCs adhered to the injured meniscus and differentiated specifically into meniscal cells. This less-invasive and targeted intra-articular delivery of ASCs may have great therapeutic potential in promoting the regeneration of meniscus or articular cartilage.

\section{Acknowledgements}

The present study was supported by the Natural Science Youth Foundation of Zhejiang Province (grant no. LQ14H060001), the Natural Science Grants of Zhejiang Province (grant no. Y210061), the Natural Science Youth Foundation of China (grant nos. 81401779, 81201414, 81201408) and the Science Technology Program of Zhejiang Province (grant no. 2013C23104).

\section{References}

1. Fairbank TJ: Knee joint changes after meniscectomy. J Bone Joint Surg Br 30B: 664-670, 1948.

2. Fox JM, Blazina ME and Carlson GJ: Multiphasic view of medial meniscectomy. Am J Sports Med 7: 161-164, 1979.

3. Jørgensen U, Sonne-Holm S, Lauridsen F and Rosenklint AL: Long-term follow-up of meniscectomy in athletes. A prospective longitudinal study. J Bone Joint Surg Br 69: 80-83, 1987.

4. Sweigart MA and Athanasiou KA: Toward tissue engineering of the knee meniscus. Tissue Eng 7: 111-129, 2001.

5. Baker P, Coggon D, Reading I, Barrett D, McLaren M and Cooper C: Sports injury, occupational physical activity, joint laxity, and meniscal damage. J Rheumatol 29: 557-563, 2002

6. Boyd KT and Myers PT: Meniscus preservation; rationale, repair techniques and results. Knee 10: 1-11, 2003.

7. Cook JL: The current status of treatment for large meniscal defects. Clin Orthop Relat Res: 88-95, 2005.

8. Setton LA, Guilak F, Hsu EW and Vail TP: Biomechanical factors in tissue engineered meniscal repair. Clin Orthop Relat Res (Suppl): S254-S272, 1999.

9. Fraser JK, Wulur I, Alfonso Z and Hedrick MH: Fat tissue: An underappreciated source of stem cells for biotechnology. Trends Biotechnol 24: 150-154, 2006.

10. Qi Y, Feng G, Huang Z and Yan W: The application of super paramagnetic iron oxide-labeled mesenchymal stem cells in cell-based therapy. Mol Biol Rep 40: 2733-2740, 2013.

11. Saldanha KJ, Doan RP, Ainslie KM, Desai TA and Majumdar S: Micrometer-sized iron oxide particle labeling of mesenchymal stem cells for magnetic resonance imaging-based monitoring of cartilage tissue engineering. Magn Reson Imaging 29: 40-49, 2011.

12. Hill JM, Dick AJ, Raman VK, Thompson RB, Yu ZX, Hinds KA, Pessanha BS, Guttman MA, Varney TR, Martin BJ, et al: Serial cardiac magnetic resonance imaging of injected mesenchymal stem cells. Circulation 108: 1009-1014, 2003.

13. An C, Cheng Y, Yuan Q and Li J: IGF-1 and BMP-2 induces differentiation of adipose-derived mesenchymal stem cells into chondrocytes-like cells. Ann Biomed Eng 38: 1647-1654, 2010.

14. Hsiao JK, Tai MF, Chu HH, Chen ST, Li H, Lai DM, Hsieh ST, Wang JL and Liu HM: Magnetic nanoparticle labeling of mesenchymal stem cells without transfection agent: Cellular behavior and capability of detection with clinical $1.5 \mathrm{~T}$ magnetic resonance at the single cell level. Magn Reson Med 58: 717-724, 2007.

15. Yang CY, Hsiao JK, Tai MF, Chen ST, Cheng HY, Wang JL and Liu HM: Direct labeling of hMSC with SPIO: The long-term influence on toxicity, chondrogenic differentiation capacity, and intracellular distribution. Mol Imaging Biol 13: 443-451, 2011.

16. Mankin HJ, Dorfman H, Lippiello L and Zarins A: Biochemical and metabolic abnormalities in articular cartilage from osteoarthritic human hips. II. Correlation of morphology with biochemical and metabolic data. J Bone Joint Surg Am 53: 523-537, 1971.

17. Pritzker KP, Gay S, Jimenez SA, Ostergaard K, Pelletier JP, Revell PA, Salter D and van den Berg WB: Osteoarthritis cartilage histopathology: Grading and staging. Osteoarthritis Cartilage 14: 13-29, 2006.

18. Henning TD, Sutton EJ, Kim A, Golovko D, Horvai A, Ackerman L, Sennino B, McDonald D, Lotz J and Daldrup-Link HE: The influence of ferucarbotran on the chondrogenesis of human mesenchymal stem cells. Contrast Media Mol Imaging 4: 165-173, 2009.

19. Arbab AS, Bashaw LA, Miller BR, Jordan EK, Lewis BK, Kalish $\mathrm{H}$ and Frank JA: Characterization of biophysical and metabolic properties of cells labeled with superparamagnetic iron oxide nanoparticles and transfection agent for cellular MR imaging. Radiology 229: 838-846, 2003.

20. Mailänder V,Lorenz MR, Holzapfel V, Musyanovych A, Fuchs K, Wiesneth M, Walther P, Landfester K and Schrezenmeier H: Carboxylated superparamagnetic iron oxide particles label cells intracellularly without transfection agents. Mol Imaging Biol 10: 138-146, 2008. 
21. Metz S, Bonaterra G, Rudelius M, Settles M, Rummeny EJ and Daldrup-Link HE: Capacity of human monocytes to phagocytose approved iron oxide MR contrast agents in vitro. Eur Radiol 14: $1851-1858,2004$

22. Qi Y, Zhao T, Xu K, Dai T and Yan W: The restoration of full-thickness cartilage defects with mesenchymal stem cells (MSCs) loaded and cross-linked bilayer collagen scaffolds on rabbit model. Mol Biol Rep 39: 1231-1237, 2012.

23. Chen FH and Tuan RS: Mesenchymal stem cells in arthritic diseases. Arthritis Res Ther 10: 223, 2008.

24. Kern S, Eichler H, Stoeve J, Klüter H and Bieback K: Comparative analysis of mesenchymal stem cells from bone marrow, umbilical cord blood, or adipose tissue. Stem Cells 24: 1294-1301, 2006.

25. Horie M, Sekiya I, Muneta T, Ichinose S, Matsumoto K, Saito H Murakami T and Kobayashi E: Intra-articular injected synovial stem cells differentiate into meniscal cells directly and promote meniscal regeneration without mobilization to distant organs in rat massive meniscal defect. Stem Cells 27: 878-887, 2009.

26. Murphy JM, Fink DJ, Hunziker EB and Barry FP: Stem cell therapy in a caprine model of osteoarthritis. Arthritis Rheum 48 3464-3474, 2003.
27. Ruiz-Ibán MA, Díaz-Heredia J, García-Gómez I, Gonzalez-Lizán F, Elías-Martín E and Abraira V: The effect of the addition of adipose-derived mesenchymal stem cells to a meniscal repair in the avascular zone: An experimental study in rabbits. Arthroscopy 27: 1688-1696, 2011.

28. Qi Y, Feng G and Yan W: Mesenchymal stem cell-based treatment for cartilage defects in osteoarthritis. Mol Biol Rep 39: 5683-5689, 2012

29. Uccelli A, Pistoia V and Moretta L: Mesenchymal stem cells: A new strategy for immunosuppression? Trends Immunol 28 : 219-226, 2007.

30. Chen X, Armstrong MA and Li G: Mesenchymal stem cells in immunoregulation. Immunol Cell Biol 84: 413-421, 2006.

31. Caplan AI and Dennis JE: Mesenchymal stem cells as trophic mediators. J Cell Biochem 98: 1076-1084, 2006.

32. Koelling S and Miosge N: Stem cell therapy for cartilage regeneration in osteoarthritis. Expert Opin Biol Ther 9: 1399-1405, 2009.

33. Bulte JW and Kraitchman DL: Iron oxide MR contrast agents for molecular and cellular imaging. NMR Biomed 17: 484-499, 2004. 